Published in final edited form as:

Curr Opin Rheumatol. 2017 May ; 29(3): 223-227. doi:10.1097/BOR.0000000000000383.

\title{
IgG4-Related Disease
}

\section{Emanuele Bozzalla Cassione, M.D. and John H. Stone, M.D., M.P.H.}

\begin{abstract}
Purpose of review-Remarkable insights have been gleaned recently with regard to the pathophysiology of IgG4-related disease (IgG4-RD). These findings have direct implications for the development of targeted strategies for the treatment of this condition.
\end{abstract}

Recent findings-Oligoclonal expansions of cells of both the B and T lymphocyte lineages are present in the blood of patients with IgG4-RD. Oligoclonal expansions of plasmablasts are a good biomarker for disease activity. An oligoclonally-expanded population of CD4+ cytotoxic T lymphocytes is found not only in the peripheral blood but also at tissue sites of active disease. This cell elaborates cytokines that may drive the fibrosis characteristic of IgG4-RD. T follicular helper cells (Tfhc), particularly the Tfhc2 subset, appear to play a major role in driving the class switch to IgG4 that typifies this disease. The relationship between malignancy and IgG4-RD remains an area of interest.

Summary-Advances in understanding the pathophysiology of IgG4-RD have proceeded swiftly, leading to the identification of a number of potential targeted treatment strategies. The completion of classification criteria for IgG4-RD, an effort supported jointly by the American College of Rheumatology and the European League Against Rheumatism, will further facilitate studies in this disease.

\section{Keywords}

IgG4-related disease; plasmablast; CD4+ cytotoxic T lymphocyte; $\mathrm{T}$ follicular helper cell

\section{Introduction}

IgG4-related disease (IgG4-RD) is a chronic fibroinflammatory condition characterized by tumefactive lesions, dense lymphoplasmacytic infiltrates, and abundant IgG4-bearing plasma cells in the affected tissues. Serum IgG4 concentrations in patients' sera are often elevated dramatically, yet are normal in approximately one third of patients with clinicopathologically-confirmed disease. IgG4-RD was described first in the pancreas - the condition once termed "lymphoplasmacytic sclerosing pancreatitis" or sometimes just "sclerosing pancreatitis", among other designations ${ }^{1}$.

Please direct correspondence to: Dr. John H. Stone, Rheumatology Clinic / Yawkey 2, Massachusetts General Hospital, 55 Fruit Street, Boston, MA. 02114, jhstone@mgh.harvard.edu, 617-726-7938.

Conflicts of interest

None. 
Common histological features are now known to characterize IgG4-RD in essentially every organ in the body ${ }^{2}$. Broader experience with this condition, however, has led to the recognition that the diagnosis is critically dependent upon careful correlation between clinical, pathological, and often radiological findings. American College of Rheumatology/ European League Against Rheumatism Classification Criteria are now being developed based on this recognition.

The immunopathogenesis of IgG4-RD remains incompletely defined. B cells at first and subsequently $\mathrm{T}$ cells have been recognized to be key players in disease pathogenesis, but their full contributions to IgG4-RD remain to be elucidated. Moreover, other elements of the immune system also likely play important roles. Treatment of IgG4-RD to date has been predicated primarily on glucocorticoids, but the growing recognition of this approach's shortcomings has spawned earlier consideration of either non-specific "disease-modifying" agents or targeted treatments, both of which are intended as steroid-sparing strategies.

\section{Role of B cells}

A first reliable advance into the pathophysiology of IgG4-RD was made when preliminary studies with rituximab (RTX) showed that B cell depletion induced disease remission and led to improvement in tissue fibrosis ${ }^{3}$. Clinical improvement was accompanied by a reduction in serum IgG4 levels. Further studies in IgG4-RD patients with active, untreated disease identified an oligoclonally-expanded population of circulating CD19+CD20$\mathrm{CD} 27+\mathrm{CD} 38+$ bright plasmablasts, cells that are the precursors of tissue-resident, antibodyproducing plasma cells). Flow cytometry studies following treatment demonstrated that clinical improvement correlated with selective depletion of this B cell subpopulation ${ }^{4}$. Many patients achieved clinical remissions without normalizing their serum $\mathrm{IgG} 4$ concentrations, even though substantial declines in serum IgG4 levels following treatment were the rule.

The B cell compartment of patients with IgG4-RD has been studied extensively because the striking serum IgG4 elevation in many patients and the abundance of IgG4+ plasma cells at sites of disease initially suggested the possibility of an underlying lymphoproliferative condition. The latter hypothesis has then been excluded because of failure to identify monoclonal plasma cells populations in the affected tissues ${ }^{2}$, but the issue of a potential relationship between malignancy and IgG4-RD risk - and vice versa - remains an important topic.

At least two lines of evidence from the humoral immune system have suggested that an antigen-driven immune response is present in IgG4-RD. Studies on the cerebrospinal fluid of subjects with IgG4-related pachymeningitis revealed the presence of oligoclonal IgG4 ${ }^{5}$. In addition, next-generation sequencing analysis on tissue biopsy samples and on the peripheral blood of IgG4-RD patients demonstrated oligoclonal expansions of somatically hypermutated $\mathrm{IgG}^{+}{ }^{+} \mathrm{B}$ cell clones ${ }^{4}$.

The oligoclonally-expanded B cells were identified by flow cytometry as being $\mathrm{CD} 19^{+} \mathrm{CD} 20^{-} \mathrm{CD} 27^{+} \mathrm{CD} 38^{+}$plasmablasts. Plasmablasts arise in germinal centres following affinity maturation from naïve CD20+ precursors. Once in the bloodstream, plasmablasts 
differentiate into antibody-secreting short- or long-lived plasma cells, accounting for the excess IgG4 production in this disease ${ }^{6}$. Plasmablast concentrations in the blood correlate well with disease activity, decreasing sharply after RTX-induced remission and reemerging during relapse. It is worth noting that the plasmablasts reemerging during disease relapses express distinct V-J repertoires compared to samples from the same patients before their initial treatment. This phenomenon, known as "clonal divergence", further supports the hypothesis of hypermutation driven by the selection of specific antigens.

\section{Role of T cells}

T cell responses have long been considered to be central to the pathophysiology of IgG4$\mathrm{RD}$, but the focus of interest within the $\mathrm{T}$ cell population has shifted within recent years. Th2 immune responses were once believed critical to IgG4-RD pathways, partly because of the high frequency of atopic symptoms observed in many patients with IgG4-RD ${ }^{7}$ and partly because of the detection of mRNA from cytokines frequently linked to Th2 responses, e.g., interleukin (IL)-4, IL-5, and IL-13 ${ }^{8}$. More recent studies, however, have demonstrated expansions of Th2 cells only in the circulation of IgG4-RD patients with an atopic history, not in those without histories of atopy ${ }^{9}$. It seems, therefore, that despite initial appearances the role of Th2 cells in IgG4-RD is marginal. Other subpopulations of T cells, however, are involved more directly in IgG4-RD pathogenesis.

A novel population of effector memory CD4+ T cells with cytotoxic function (CD4+ CTLs) has then been described in IgG4-RD patients ${ }^{10}$. Substantial evidence suggests that this population of cells plays an important role in the pathophysiology of this disease. CD4+ CTLs are expanded in both the peripheral blood and in affected organs of IgG4-RD patients. Moreover, together with circulating plasmablasts (though at a slower rate), these CD4+CTLs decline following RTX treatment, supporting the concept that cells of the B and T cell lineages cooperate closely in mediating this condition ${ }^{10}$.

Cytotoxicity associated with CD4+ T helper lymphocytes is a concept that has emerged progressively in the past few years. The concept contrasts with the traditional view that cytotoxic T cells arise only from MHC class I restricted CD8+ T lymphocytes. During thymic development, lineage commitment towards $\mathrm{CD} 4+$ or $\mathrm{CD} 8+\mathrm{T}$ cell fate is driven by the action and counteraction of the key transcription factors ThPOK and Runx3, respectively ${ }^{11}$. CD4+CTLs seem to represent highly differentiated, antigen-experienced (memory) $\mathrm{T}$ cells with features of both $\mathrm{CD} 4+$ and $\mathrm{CD} 8+\mathrm{T}$ lymphocytes (though there are CD8- $)^{12}$. It is likely that this cell population arises from chronic antigenic stimulation. Indeed, in response to repeated antigenic stimulation, ThPOK is downregulated, resulting in a cytolytic gene expression program in activated CD4+ T cells, with differentiation into MHC class II-restricted CD4+ CTLs and an effector cell phenotype ${ }^{11}$.

The CD4+ CTLs identified express high levels of CD11a and CD11b integrins, and CD45 isoforms -RO and -RB. They lack the costimulatory receptors CD27, CD28, the chemokine receptor CCR7, and CD45-RA. Small numbers of CD4+ CTLs can be detected in the blood of healthy individuals ${ }^{13}$, and they significantly increase during chronic viral infections (such as cytomegalovirus ${ }^{14}$, Epstein-Barr virus ${ }^{15}$, human immunodeficiency virus ${ }^{13}$ ), 
malignancies ${ }^{16}$, and autoimmune disorders ${ }^{17}$. CD4+ CTLs seem to bear protective functions such as control of infected cells or elimination of transformed cells, thanks to their cytolytic MHC class II restricted action. Nevertheless, the accumulation of CD4+ CTLs in the setting of autoimmune conditions such as rheumatoid arthritis ${ }^{18}$ and inflammatory bowel disease ${ }^{19}$ suggests that these cells might also contribute to chronic inflammation. Indeed, CD4+ CTLs numbers correlate with disease severity in rheumatoid arthritis and ankylosing spondylitis, and decrease after treatment with anti-TNFalpha agents ${ }^{20}$.

In summary, the CD4+ CTLs identified in the context of IgG4-RD represent the most carefully phenotyped such cell studied to date. The cell appears to have significant potential to contribute to chronic inflammation of a variety of forms.

\section{Role of T follicular helper cells}

The powerful evidence of class-switching in IgG4-RD has led to substantial interest in the role of $\mathrm{T}$ follicular helper ( $\mathrm{Tfh}$ ) cells in this condition. Tfh cells are known to be involved in the differentiation of B cells during their development, and to contribute significantly to class switching ${ }^{21}$. Akiyama et al. ${ }^{22-23}$ have reported that among Tfh cells subsets, Tfh 2 cells induce the differentiation of naïve B cells into plasmablasts, subsequently promoting the production of IgG4 in active, untreated IgG4-RD. Circulating Tfh2 cells are expanded IgG4$\mathrm{RD}$ and their concentrations are linked to disease activity, the concentrations of circulating plasmablasts, and serum IgG4 levels.

In contrast, although circulating activated Tfh1 cells were also found to be expanded in IgG4-RD, their levels correlated with disease activity but not with serum IgG4 levels. These findings support the hypothesis of a greater role for Tfh 2 cells in the class switch observed in IgG4-RD. It is known that Tfh cells in germinal centers cooperate with B cells in the formation and antibody production. Therapy with glucocorticoids did not affect $\mathrm{Tfh} 2$ cell counts, but did result in a decrease in numbers of plasmablasts and levels of serum IgG4 and IL-4. Because IL-4 is believed to be produced by Tfh 2 cells and not by the other Tfh cell subsets, it is possible that glucocorticoid treatment affects the function and not the number of these cells.

\section{Plasmacytoid dendritic cells}

Plasmacytoid dendritic cells (pDCs) were shown to be important in the development of pancreatic inflammation through production of IFN-alpha ${ }^{24}$. Autoimmune pancreatitis (AIP) in IgG4-RD patients is associated with an infiltration of pDCs. Peripheral pDCs from IgG4$\mathrm{RD}$ patients were shown to enhance IgG4 antibody production by cells through IFN-alpha mediated signaling. Demonstration of a role for IFN-alpha in this disease comes from studies conducted on experimental AIP models in which regression of the inflammation was seen to occur with depletion of IFN-alpha production or signaling.

Final proof of its relevance in AIP in humans requires studies with pDC-depleting antibodies $(\mathrm{Ab})$ or neutralizing IFN-alpha receptor antibodies. In addition to pDCs, inflamed pancreata also harbor NETs. These structures have been seen to be involved in the activation of $\mathrm{pDCs}^{25-26}$. In fact, co-cultures of pDCs and neutrophils, forced to express NETs by 
monosodium urate (MSU) crystals or anti-lacto-ferrin (LF) Ab (NET component protein) showed increased production of IFN-alpha and the B lymphocyte activating factor known as BAFF. Moreover, when these cells were co-cultured together with B cells, they led to increases in IgG4 production.

Some data do support the notion that anti-LF Ab contribute to IgG4-RD. Serum anti-LF Ab titers, especially those of the IgG4 subclass, were elevated in the serum of IgG4-RD patients. In contrast, there has to date been no demonstration of MSU deposition in the IgG4-related pancreatic lesion.

\section{Therapy}

A group of international experts published a Consensus Guidance Statement on the Management of IgG4-RD ${ }^{27}$. This effort grew out of the Second International Symposium on IgG4-RD and Associated Conditions, held in 2014. The Third such Symposium is scheduled for 2017 and it is there that the ACR/EULAR Classification Criteria will be completed.

RTX was used initially in patients who did not respond to glucocorticoids, conventional steroid-sparing agents, or both, under the assumption that B-cell depletion might ameliorate the condition through decreasing serum IgG4 concentrations ${ }^{28}$. The fundamental assumption underlying this approach now seems not entirely true. Indeed, careful mechanistic studies of patients with IgG4- RD treated with RTX have led to novel insights about the pathophysiology of this disorder. First, B-cell depletion targets the subset of plasma cells that produce $\mathrm{IgG} 4$ in $\mathrm{IgG} 4-\mathrm{RD}^{29}$, by depleting all circulating $\mathrm{CD} 20$ - positive $\mathrm{B}$ cells, the precursors of short-lived plasma cells. Second, IgG4+ plasmablasts (CD38+CD27+CD19+CD20-IgG4+ cells) seem to be a good biomarker for IgG4-RD and are superior to serum IgG4 concentrations for diagnosis and monitoring of disease activity ${ }^{30}$.

Yamamoto et al. ${ }^{31}$ described the use of abatacept (CTLA4-Ig) to treat one patient with IgG4$\mathrm{RD}$ whose condition had been refractory to RTX. Given the recognition of the importance of $\mathrm{T}$ cells in this disease now emerging, greater attention to treatment strategies directly targeting $\mathrm{T}$ cell function may be of value.

\section{Tumor association}

Discussions of a possible link between IgG4-RD and malignancy have emerged recently. Evidence against such a relationship, however, came from a retrospective study showed that history of malignancy was 2.5 times more likely in IgG4-RD patients compared to the general US population ${ }^{32}$. Morever, a history of malignancy was three times more common among IgG4-RD patients than among control patients in a case-control analysis performed as part of the same study. Prostate cancer was the most common malignancy in both the IgG4-RD patients and the controls, a point not surprising given the demographics of IgG4$\mathrm{RD}$ and its tendency to afflict middle-aged to elderly males. It is worth noting, however, that lymphoma was responsible for $19 \%$ of the malignancies in the IgG4-RD cohort, compared to only $4 \%$ in the control cohort. This data may suggest a possible interplay between the immune dysregulation found in IgG4-RD and lymphomagenesis. 
Disease history of IgG4-RD seems to differ between IgG4-RD patients with malignancy and those without. IgG4-RD patients with malignancy history develop IgG4-RD at a later age and had higher serum IgG4 concentrations compared to the subgroup without malignancy. It should be noted that no cases of IgG4-RD involving the organ previously affected by cancer were reported.

Malignancy may therefore be a predisposing condition, at least in some patients. One hypothesis is that treatment of malignancy (e.g., radiation, chemotherapy) favors immune deregulation. Alternatively, there might be a common genetic predisposing background behind both malignancy and IgG4-RD. However, the fact that no IgG4-RD manifestation occurred at the site of a prior malignancy suggests that local changes deriving from the tumor itself or from its treatment are not a likely explanation. We anticipate further discussion about the potential connections between IgG4-RD and malignancies in the future.

\section{Conclusion}

The large increase in literature production on IgG4-RD in these past years reflects the increasing interest of scientific community about this topic. Despite the increased efforts, the exact pathophysiology standing behind this fibroinflammatory condition still remains enigmatic. Studies in the last years, highlighting a role for T cell subpopulations, namely CD4+ CTLs and Tfh cells, as well as plasmablasts and pDCs in IgG4-RD, seem to open new vistas from which to explore further the biological processes leading to fibrosis. We anticipate increasing interest in targeted disease therapies based on emerging pathophysiological insights.

\section{Acknowledgments}

Financial Support \& Sponsorship

Dr. Stone is the Edward A. Fox Chair in Medicine at the Massachusetts General Hospital. This work was also funded in part by the Shankaran \& Vyoma Nair Fund for IgG4-Related Disease Research and the Massachusetts General Hospital Autoimmune Disease Center of Excellence (ACE) for IgG4-RD (AI 110495; P.I.: Shiv Pillai).

\section{References}

1. Hamano H, Kawa S, Horiuchi a, et al. High serum IgG4 concentrations in patients with sclerosing pancreatitis. N Engl J Med. 2001; 344(10):732-738. DOI: 10.1056/NEJM200103083441005 [PubMed: 11236777]

2. Deshpande V, Zen Y, Chan JK, et al. Consensus statement on the pathology of IgG4-related disease. Mod Pathol. 2012; 25(9):1181-1192. DOI: 10.1038/modpathol.2012.72 [PubMed: 22596100]

3*. Della-Torre E, Feeney E, Deshpande V, et al. B-cell depletion attenuates serological biomarkers of fibrosis and myofibroblast activation in IgG4-related disease. Ann Rheum Dis. 2015; 74(12): 2236-2243. Reports an unanticipated downstream effect of B cell depletion: a positive impact on fibrosis. DOI: 10.1136/annrheumdis-2014-205799 [PubMed: 25143523]

4**. Mattoo H, Mahajan VS, Della-Torre E, et al. De novo oligoclonal expansions of circulating plasmablasts in active and relapsing IgG4-related disease. J Allergy Clin Immunol. 2014; 134(3): 679-687. Identifies plasmablasts as a crucial player in IgG4-RD and reports the finding of clonal divergence, implicating an antigen-driven process. DOI: 10.1016/j.jaci.2014.03.034 [PubMed: 24815737] 
5. Della Torre E, Bozzolo EP, Passerini G, Doglioni C, Sabbadini MG. IgG4-related pachymeningitis: evidence of intrathecal IgG4 on cerebrospinal fluid analysis. Ann Intern Med. 2012; 156(5):401403. DOI: 10.7326/0003-4819-156-5-201203060-00025 [PubMed: 22393144]

6. Hiepe F, Dörner T, Hauser AE, Hoyer BF, Mei H, Radbruch A. Long-lived autoreactive plasma cells drive persistent autoimmune inflammation. Nat Rev Rheumatol. 2011; 7(3):170-178. DOI: 10.1038/ nrrheum.2011.1 [PubMed: 21283146]

7. Della Torre E, Mattoo H, Mahajan VS, Carruthers M, Pillai S, Stone JH. Prevalence of atopy, eosinophilia, and IgE elevation in IgG4-related disease. Allergy Eur J Allergy Clin Immunol. 2014; 69(2):269-272. DOI: 10.1111/all.12320

8. Kanari H, Kagami SI, Kashiwakuma D, et al. Role of Th2 cells in IgG4-related lacrimal gland enlargement. International Archives of Allergy and Immunology. 2010; 152:47-53. DOI: 10.1159/000312125 [PubMed: 20523063]

9*. Mattoo H, Della-Torre E, Mahajan VS, Stone JH, Pillai S. Circulating Th2 memory cells in IgG4related disease are restricted to a defined subset of subjects with atopy. Allergy Eur J Allergy Clin Immunol. 2014; 69(3):399-402. Debunks the notion that the Th2 pathway is central to IgG4-RD. DOI: 10.1111/all.12342

10**. Mattoo H, Mahajan VS, Maehara T, et al. Clonal expansion of CD4+ cytotoxic T lymphocytes in patients with IgG4-related disease. J Allergy Clin Immunol. 2016; 138(3):825-838. Describes in detail the phenotype of the cell regarded as the linchpin of IgG4-RD. DOI: 10.1016/j.jaci. 2015.12.1330 [PubMed: 26971690]

11. Cheroutre H, Husain MM. CD4 CTL: Living up to the challenge. Semin Immunol. 2013; 25(4): 273-281. DOI: 10.1016/j.smim.2013.10.022 [PubMed: 24246226]

12. Hintzen RQ, de Jong R, Lens SM, Brouwer M, Baars P, van Lier Ra. Regulation of CD27 expression on subsets of mature T-lymphocytes. J Immunol. 1993; 151(5):2426-2435. [PubMed: 7689607]

13. Appay V, Zaunders JJ, Papagno L, et al. Characterization of CD4(+) CTLs ex vivo. J Immunol. 2002; 168(11):5954-5958. DOI: 10.4049/jimmunol.168.11.5954 [PubMed: 12023402]

14. Casazza JP, Betts MR, Price Da, et al. Acquisition of direct antiviral effector functions by CMVspecific CD4+ T lymphocytes with cellular maturation. J Exp Med. 2006; 203(13):2865-2877. DOI: 10.1084/jem.20052246 [PubMed: 17158960]

15. Haigh TaLin X, Jia H. , et al. EBV latent membrane proteins (LMPs) 1 and 2 as immunotherapeutic targets: LMP-specific CD4+ cytotoxic T cell recognition of EBV-transformed B cell lines. J Immunol. 2008; 180(3):1643-1654. DOI: 10.4049/jimmunol.180.3.1643 [PubMed: 18209060]

16. Martorelli D, Muraro E, Merlo A, Turrini R, Rosato A, Dolcetti R. Role of CD4+ cytotoxic T lymphocytes in the control of viral diseases and cancer. Int Rev Immunol. 2010; 29(4):371-402. DOI: 10.3109/08830185.2010.489658 [PubMed: 20635880]

17. Broux B, Markovic-Plese S, Stinissen P, Hellings N. Pathogenic features of CD4+CD28- T cells in immune disorders. Trends Mol Med. 2012; 18(8):446-453. DOI: 10.1016/j.molmed.2012.06.003 [PubMed: 22784556]

18. Thewissen M, Somers V, Hellings N, Fraussen J, Damoiseaux J, Stinissen P. CD4+CD28null T cells in autoimmune disease: pathogenic features and decreased susceptibility to immunoregulation. J Immunol. 2007; 179:6514-6523. DOI: 10.1016/j.clim.2007.03.058 [PubMed: 17982040]

19. Kobayashi T, Okamoto S, Iwakami Y, et al. Exclusive increase of CX3CR1+CD28-CD4+ T cells in inflammatory bowel disease and their recruitment as intraepithelial lymphocytes. Inflamm Bowel Dis. 2007; 13(7):837-846. DOI: 10.1002/ibd.20113 [PubMed: 17285595]

20. Gerli R, Schillaci G, Giordano A, et al. CD4+CD28- T lymphocytes contribute to early atherosclerotic damage in rheumatoid arthritis patients. Circulation. 2004; 109(22):2744-2748. DOI: 10.1161/01.CIR.0000131450.66017.B3 [PubMed: 15159291]

21. McHeyzer-Williams LJ, Pelletier N, Mark L, Fazilleau N, McHeyzer-Williams MG. Follicular helper T cells as cognate regulators of B cell immunity. Curr Opin Immunol. 2009; 21(3):266-273. DOI: 10.1016/j.coi.2009.05.010 [PubMed: 19502021] 
22. Akiyama M, Suzuki K, Yamaoka K, et al. Number of circulating follicular helper $2 \mathrm{~T}$ cells correlates with IgG4 and interleukin-4 levels and plasmablast numbers in IgG4-related disease. Arthritis Rheumatol. 2015; 67(9):2476-2481. DOI: 10.1002/art.39209 [PubMed: 25989153]

23**. Akiyama M, Yasuoka H, Yamaoka K, et al. Enhanced IgG4 production by follicular helper $2 \mathrm{~T}$ cells and the involvement of follicular helper $1 \mathrm{~T}$ cells in the pathogenesis of IgG4-related disease. Arthritis Res Ther. 2016; :1-14. Implicate TFHC2 as the driver of the class switch in IgG4-RD. DOI: 10.1186/s13075-016-1064-4 [PubMed: 26732314]

24**. Arai Y, Yamashita K, Kuriyama K, et al. Plasmacytoid Dendritic Cell Activation and IFN-a Production Are Prominent Features of Murine Autoimmune Pancreatitis and Human IgG4Related Autoimmune Pancreatitis. J Immunol. 2015; 195(7):3033-3044. Raises the intriguing possibility that plasmacytoid dendritic cells play a role in IgG4-related AIP. DOI: 10.4049 / jimmunol.1500971 [PubMed: 26297761]

25. Lande R, Ganguly D, Facchinetti V, et al. Neutrophils Activate Plasmacytoid Dendritic Cells by Releasing Self-DNA-Peptide Complexes in Systemic Lupus Erythematosus. Sci Transl Med. 2011; 3(73):73ra19.doi: 10.1126/scitranslmed.3001180

26. Döring Y, Manthey HD, Drechsler M, et al. Auto-antigenic protein-DNA complexes stimulate plasmacytoid dendritic cells to promote atherosclerosis. Circulation. 2012; 125(13):1673-1683. DOI: 10.1161/CIRCULATIONAHA.111.046755 [PubMed: 22388324]

27. Khosroshahi A, Wallace ZS, Crowe JL, et al. International consensus guidance statement on the management and treatment of IgG4-related disease. Arthritis Rheumatol (Hoboken, NJ). 2015; 67(7):1688-1699. DOI: 10.1002/art.39132

28. Khosroshahi A, Bloch DB, Deshpande V, Stone JH. Rituximab therapy leads to rapid decline of serum IgG4 levels and prompt clinical improvement in IgG4-related systemic disease. Arthritis Rheum. 2010; 62(6):1755-1762. DOI: 10.1002/art.27435 [PubMed: 20191576]

29. Khosroshahi A, Carruthers MN, Deshpande V, Unizony S, Bloch DB, Stone JH. Rituximab for the treatment of IgG4-related disease: Lessons from 10 consecutive patients. Medicine (Baltimore). 2012; 91:57-66. DOI: 10.1097/MD.0b013e3182431ef6 [PubMed: 22210556]

30*. Wallace ZS, Mattoo H, Carruthers M, et al. Plasmablasts as a biomarker for IgG4-related disease, independent of serum IgG4 concentrations. Ann Rheum Dis. 2015; 74:190-195. Reports the correlation of blood plasmablast concentratons and disease activity. DOI: 10.1136/ annrheumdis-2014-205233 [PubMed: 24817416]

31. First ARDO. Could abatacept directly target expanded plasmablasts in IgG4-related disease? 2016; 0(0):19-21. DOI: 10.1136/annrheumdis-2016-16

32*. Wallace ZS, Wallace CJ, Lu N, Choi HK, Stone JH. Association of IgG4-Related Disease With History of Malignancy. Arthritis Rheumatol. 2016; 68(9):2283-2289. Calls into question the idea that IgG4-RD is a precursor of malignancy. In fact, the opposite may be true. DOI: $10.1002 /$ art. 39773 [PubMed: 27273903] 


\section{Key Points}

- Oligoclonal expansions of cells of both the B and T lymphocyte lineages are present in the blood of patients with IgG4-RD.

- Blood plasmablast concentrations correlate well with disease activity - better even than do serum IgG4 levels.

- An oligoclonally-expanded population of CD4+ cytotoxic T lymphocytes, found not only in the peripheral blood but also at tissue sites of active disease, elaborates cytokines that may drive the fibrosis characteristic of IgG4-RD.

- $\quad$ TFHC2 appear to play a major role in driving the IgG4 class switch.

- Data from a recent study suggest that a history of malignancy may be a risk factor for IgG4-RD rather than vice versa.

- The completion of classification criteria for IgG4-RD, an effort supported jointly by the American College of Rheumatology and the European League Against Rheumatism, will further facilitate studies in this disease. 